\title{
Isotherms Determined by the National Bureau of Standards Acoustical Thermometer in the Liquid Helium Temperature Range
}

\author{
G. Cataland and H. Plumb
}

\begin{abstract}
(July 7, 1965)
Isotherms of the speed of sound in helium 4 gas as a function of pressure have been measured. Five of the isotherms in the liquid helium 4 temperature range have been analyzed, and the results are discussed. The acoustically determined values of temperature are consistently higher than the values of temperature associated with the liquid helium 4 vapor pressure scale $\left(\mathrm{T}_{58}\right)$. The deviations are $0.006{ }^{\circ} \mathrm{K}$ at $2.323{ }^{\circ} \mathrm{K}, 0.007{ }^{\circ} \mathrm{K}$ at $2.807{ }^{\circ} \mathrm{K}, 0.008{ }^{\circ} \mathrm{K}$ at $3.211{ }^{\circ} \mathrm{K}, 0.010{ }^{\circ} \mathrm{K}$ at $4.212{ }^{\circ} \mathrm{K}$ and $0.012{ }^{\circ} \mathrm{K}$ at $5.024{ }^{\circ} \mathrm{K}$.
\end{abstract}

Key Words: Acoustical thermometer, isotherm at low temperature, Speed of sound in helium gas, thermometry at low temperature.

\section{Introduction}

A program of absolute and secondary low temperature thermometry has been conducted for some years at the National Bureau of Standards. Certain of the results are in the process of being reported and this paper presents absolute temperature determinations in the lower range of investigation.

Thus far, absolute thermometry at these temperatures has been conducted by employing either a gas thermometer or apparatus designed for P - V isotherm determinations. Each of these methods is subject to inaccuracies which require corrections in order to obtain "the thermodynamic temperature." The corrections are caused by or related to "dead space volumes", deviations of the thermometric fluid from "ideal gas", behavior, conservation of the thermometric fluid during measurements, and volume changes accompanying necessary temperature changes. In some cases the pressure measurements, the requirements of which are far from being trivial, must be modified to allow for thermomolecular effects. To some extent, these corrections have been considered as existing nuisances but not always necessarily crucial in determining the end result, a temperature. While it is not the intention of this paper to treat the preceding corrections, the investigation being reported was initiated with an intention of avoiding them. Additionally we felt that a fundamentally different approach to low temperature thermometry was completely justified and would prove to be beneficial in contributing knowledge about existing temperature determinations and scales.

A salient feature of gas thermometry should be mentioned. The thermometer must be filled with the thermometric gas and the pressure measured at some assumed value of temperature. This assumption is critical and receives careful consideration, naturally, but a cascading of errors from one temperature range to another is possible. This type of error is also avoided in our approach to thermometry.

The method of temperature determinations which we have pursued is based on the speed of sound in helium gas as a function of the gas pressure. Since translational dispersion of speeds in our experiments is negligible, one can proceed from the basic equation $W=\sqrt{E / \rho}$ where $W$ is the speed of sound:

$$
E \text {, the adiabatic bulk }
$$

modulus of the medium; and $\rho$ the density. This can be written as

$$
W=\sqrt{-\frac{v^{2}}{M}\left(\frac{\delta p}{\delta v}\right)_{S}}=\sqrt{-\frac{C_{p}}{C_{v}} \frac{v^{2}}{M}\left(\frac{\delta p}{\delta v}\right)_{T}} .
$$

If the equation of state for helium gas is assumed to be of the form

$$
p v=R T\left(1+\frac{B}{v}+\frac{C}{v^{2}}+\ldots\right)
$$

the partial derivative, $\left(\frac{\delta p}{\delta v}\right)_{T}$, can be substituted into eq (2) and $C_{p} / C_{v}$ can be related to an ideal gas with the result

$$
W^{2}=\left(\frac{C_{p}}{C_{v}}\right)_{p=0} \frac{R_{M} T}{M_{\mathrm{He}}}\left(1+\alpha p+\beta p^{2}+\ldots . .\right) .
$$


In eq (4), $T$ is the desired temperature;

$$
\begin{aligned}
\left(\frac{C_{p}}{C_{v}}\right)_{p=0}=5 / 3 ; R_{M}=8.314 \times 10^{7}\left(\mathrm{erg} /{ }^{\circ} \mathrm{K} \mathrm{mol}\right) ;{ }^{1} \\
M_{\mathrm{He}}=4.0026 \mathrm{~g} / \mathrm{mol}[2] ;{ }^{2}
\end{aligned}
$$

and $\alpha=\frac{1}{R T}\left(2 B+\frac{4}{3} T \frac{d B}{d T}+\frac{4}{15} T^{2} \frac{d^{2} B}{d T^{2}}\right) . \quad B$ and $C$ are the second and third virial coefficients, respectively, for helium 4 gas. Equation (4) suggests the form

$$
\begin{aligned}
& W^{2}=W_{0}^{2}\left(1+\alpha p+\beta p^{2}+\ldots\right), \\
& \text { where } W_{0}^{2}=\left(\frac{C_{p}}{C_{v}}\right)_{p=0} \frac{R_{M} T}{M_{\mathrm{He}}}
\end{aligned}
$$

in which $W_{0}$ is the speed of sound in an ideal gas. $W$ and $p$ are measured experimentally and treated so that $W_{0}^{2}$ may be determined. In practice this may be done in two ways. $W^{2}$ and $p$ as in eq (5) may be fitted by a least squares method with the intercept $W_{0}^{2}$, at $p=0$ resulting. Another approach is to obtain $W_{0}$ or $W_{0}^{2}$ from a linear extrapolation (either graphically or analytically). The implication is, naturally, that in eq (5) $\alpha p$ is small compared with 1 , and terms involving higher orders of pressure negligible. Values of $W_{0}^{2}$ obtained by the methods described afford a simple calculation of the temperature. Obviously, this treatment can produce information on helium 4 virial coefficients, but this will be presented in a following paper.

The experimentally determined quantity, speed, has been obtained with an apparatus which is generally called an acoustical interferometer [3]. This instrument employs a quartz crystal, operating at its resonant frequency (1 megahertz), and it is assumed that the crystal acts as a plane wave radiator. Pressure measurements are made with a mercury manometer which is accurate to $70 \mu$ of mercury in the range of measurements. While there are a few references to our instrument in the literature $[4,5,6]$, a detailed description has not been published (see footnote 3 ).

\section{Results}

There are listed in table 1 values of pressure, speed and speed squared for each isotherm. The temperature of an "isotherm" was maintained nearly constant by an automatic regulator which operated from a germanium resistor connected to a Wheatstone bridge. This and other germanium resistors were located in thermal contact with the acoustical thermometer apparatus so that allowance could be made for small relative temperature changes which occurred during measurements of an isotherm. Since four or five pressure-speed determinations on a given isotherm

\footnotetext{
1 The value of the gas constant is taken from reference [1]

${ }^{2}$ Figures in brackets indicate the literature references at the end of this paper.

${ }^{3}$ Note added in proof: The instrument is described in Science 150, No. 3693, p. 155 (8 Oct. 1965 .
}

require a day it was not possible for all points on an isotherm to be taken at exactly the same temperature. Consequently, small deviations in temperature occurred (not exceeding $3 \mathrm{mdeg}$ ) and the speeds were corrected using the germanium thermometer indications. The speeds in table 1 have been treated in this manner.

TABLE 1. Isotherm data (sneed-meters/sec; pressureatmospheres)

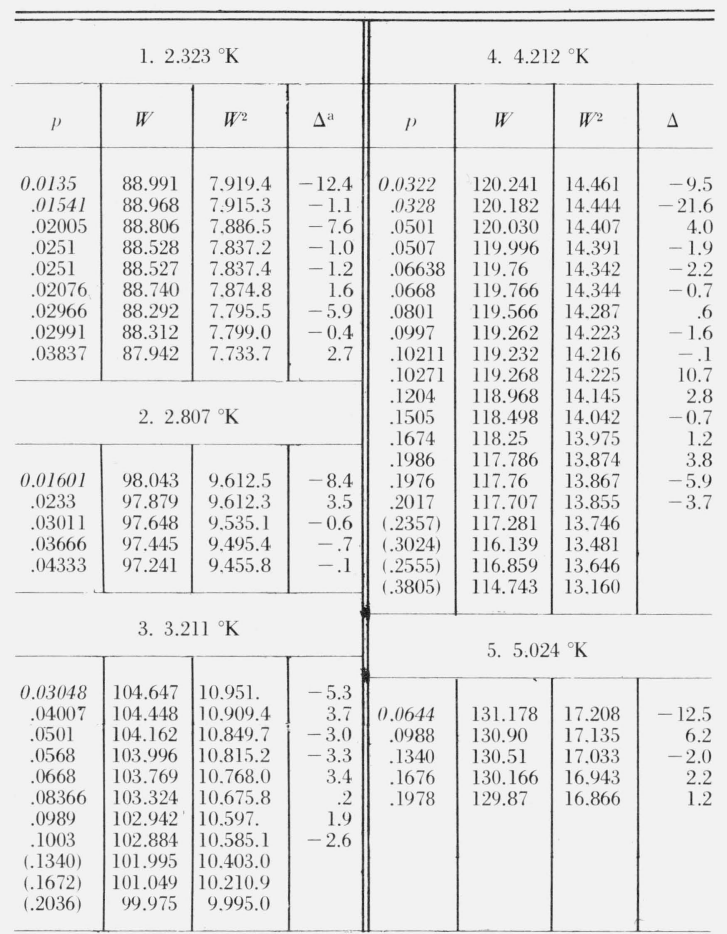

a The columns under $\Delta$ contain the difference between the experimental $W^{2}$ at the indicated pressures and the $W^{2}$ of linear function determined by the computer least squares fitting.

For each isotherm, values of $p$ and $W^{2}$ have been treated analytically by computer and graphically to obtain $W_{0}^{2}$. Equation (5) demonstrates that a linear treatment of the data will be inadequate at sufficiently high pressures. It is not surprising, therefore, that in figure 1 , which depicts the $3.2^{\circ} \mathrm{K}$ isotherm, the data points markedly depart from a straight line above 0.17 atm of pressure. In order to represent the isotherms linearly, we noted the departure of the highest point and, assuming that such departures are proportional to $p^{2}$, discarded all points for which the deviation from linearity would exceed the experimental reproducibilities. Accordingly, the data above 0.1003 and 0.2017 atm for isotherms 3.2 and 4.2 ${ }^{\circ} \mathrm{K}$, respectively, were omitted in the linear computer analysis. For the remaining isotherms, comparable data selection was unnecessary since correspondingly high pressure data had not been acquired.

In general, the lowest pressure points on an isotherm warrant lesser weightings. The inaccuracy of these 


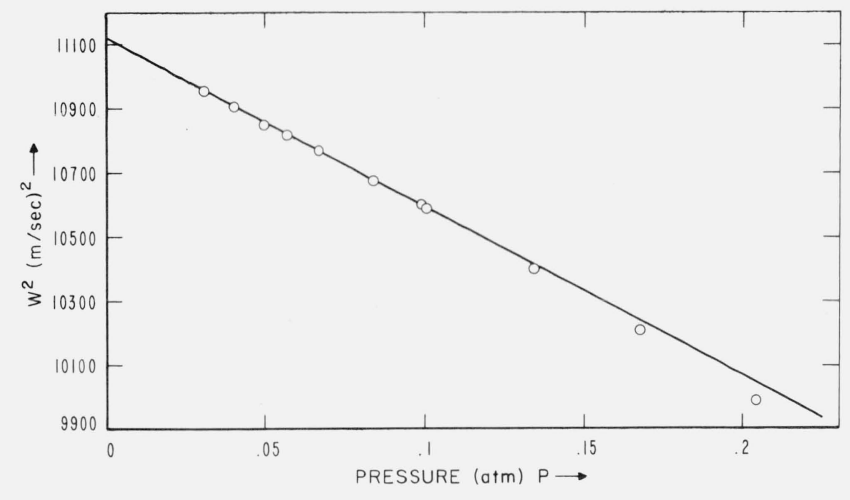

Figure 1. $\mathrm{W}^{2}$ as a function of pressure at $3.211^{\circ} \mathrm{K}$.

points is caused by a lack of resolution resulting from the high attenuation of sound energy in the thermometric gas. Consequently, the italicized data received one quarter weights in all computer fits.

Column 1 of table 2 contains the $W_{0}^{2}$ intercepts obtained from graphical treatment of the data where $W^{2}$ was plotted as a function of $p$. The procedure was straightforward in that straight lines were obvious representations in the graphs if the highest pressure points for the 3.2 and $4.2{ }^{\circ} \mathrm{K}$ isotherms were not considered. Column 8 of table 2 contains the temperatures derived from the $W_{0}^{2}$ 's of column 1 .

In the computer analysis of the apparently linear data, each isotherm was fitted by the method of least squares to a polynomial in which the square of the speed is equated to a power series of the pressure, i.e., $W^{2}=W_{0}^{2}+W_{0}^{2} \alpha p+W_{0}^{2} \beta p^{2}+W_{0}^{2} \gamma p^{3}+\ldots$. The first analysis involved the constant and linear terms, the second included the quadratic, and for the third the cubic was also added. Each of these cases yields a value for $W_{0}^{2}$. Columns 2 and 9 of table 2 list the intercepts and temperatures obtained from the linear computer fitting. The higher order fits, with one exception, proved to be of little significance since the additional coefficients were statistically uncertain. The exception was the quadratic fit for the $4.2{ }^{\circ} \mathrm{K}$ isotherm. In this case a $W_{0}^{2}$ value of 14,570 with an uncertainty of 6 was obtained from which a temperature of $4.209( \pm 0.002){ }^{\circ} \mathrm{K}$ resulted. ${ }^{4}$ In the above analyses the bracketed high pressure data points for the 3.2 and $4.2{ }^{\circ} \mathrm{K}$ were not included.

Equation (5) can be accurately represented by a linear expression (for a constant $\alpha$ ) if the pressure is sufficiently low. (Our input data for the majority of isotherms have been selected to meet this criterion or, more specifically, data have been used that graphically exhibit a linear relationship within the limits of the experimental error.) For this reason we believe that the most accurate determinations of isotherm temperatures are those which have resulted from a linear treatment. Nevertheless for completeness, it is necessary to extend the computer analysis to higher orders of pressure and include the higher pressure data for isotherms 3.2 and $4.2^{\circ} \mathrm{K}$. In this case the linear representation is inaccurate, but there exists no a priori approach to predict the significance of particular higher order pressure terms since the analytical representation of higher order virial coefficients is unknown. Consequently we have approached the analyses empirically, by including quadratic, and quadratic and cubic terms for the pressure.

When only the quadratic term is added, a statistically meaningful coefficient for $p^{2}$ of eq (5) results. Columns 5 and 11 list the $W_{0}^{2}$ and $T$ values which result from extrapolations of the quadratic analyses. The addition of a cubic term was not helpful since the

${ }^{4}$ The values reported here are not to be confused with the value resulting from the more complete quadratic fitting as reported in columns 5. 6, and 7 of table 2.

TABLE 2. Summary of data derived from isotherms

\begin{tabular}{|c|c|c|c|c|c|c|c|c|c|c|c|c|}
\hline \multicolumn{7}{|c|}{$W^{2}$ Extrapolations from } & \multicolumn{5}{|c|}{ Temperatures derived from } & \multirow{4}{*}{$\begin{array}{c}T_{\text {isotherm }}-T_{5 x} \\
\Delta T\end{array}$} \\
\hline \multicolumn{4}{|c|}{ Linear analysis of data } & \multirow{2}{*}{\multicolumn{3}{|c|}{$\begin{array}{c}\text { Quadratic analysis } \\
\text { of data } \\
\text { Computer }\end{array}$}} & \multicolumn{3}{|c|}{ Linear analysis } & \multirow{2}{*}{\multicolumn{2}{|c|}{$\begin{array}{c}\begin{array}{c}\text { Quadratic } \\
\text { Analysis }\end{array} \\
\text { Computer }\end{array}$}} & \\
\hline Graphical & Cor & puter & & & & & Graphical & Computer & Computer & & & \\
\hline$W_{0}^{2}$ & $W_{0}^{\prime 2}$ & $\delta^{\mathrm{a}}$ & $\sigma^{c}$ & $W_{0}^{2}$ & $\delta^{\mathrm{a}}$ & $\sigma^{e}$ & $T$ & $T$ & $\delta T^{\mathrm{b}}$ & $T$ & $\delta T^{\mathrm{b}}$ & \\
\hline Column No. 1 & 2 & 3 & 4 & 5 & 6 & 7 & 8 & 9 & 10 & 11 & 12 & 13 \\
\hline 17.393 & 17.392 & 9.6 & 5.4 & & & & 5.024 & 5.024 & 0.002 & & & 0.012 \\
\hline 14.580 & 14.583 & 3.1 & 5.0 & 14.568 & 6. & 7.2 & 4.211 & 4.212 & .001 & 4.208 & 0.002 & .010 \\
\hline 11.123 & 11.117 & 3.7 & 3.1 & 11.104 & 4.4 & 2.9 & 3.213 & 3.211 & .001 & 3.207 & .001 & .008 \\
\hline 9.722 .5 & 9.717 .5 & 6.2 & 3.1 & & & & 2.808 & 2.807 & .002 & & & .007 \\
\hline 8.037 .5 & 8.040 .5 & 6.9 & 4.5 & & & & 2.322 & 2.323 & .002 & & & .006 \\
\hline
\end{tabular}


statistical significance of coefficients was lost. The analysis was not extended for we felt that to be meaningful it should include data over the largest possible range of pressures. Column 13 contains the temperature differences, $T_{\text {isotherm }}-T_{58}$, where $T_{58}$ is derived from the helium 4 vapor pressure scale [7]. These were obtained by calibrating germanium resistors directly against measured vapor pressures and comparing them, in the acoustical thermometer, with germanium resistors which had been calibrated by the acoustical thermometer. In one or two cases, a resistor associated with the acoustical thermometer during an isotherm measurement had been previously calibrated at one or more vapor pressure temperatures. The temperature values of $T_{\text {isotherm }}$ are from column 9 .

Thus our values of isotherm temperatures, column 9 , and the indicated departures from $T_{58}$ (column 13) afford a comparison with the $T_{58}$ scale as portayed in figure 2. Except for out data points, figure 2 is taken from Van Dijk [8]. In essence he has plotted values of absolute temperature determination, in the liquid helium range, relative to the $T_{58}$ scale. While we are

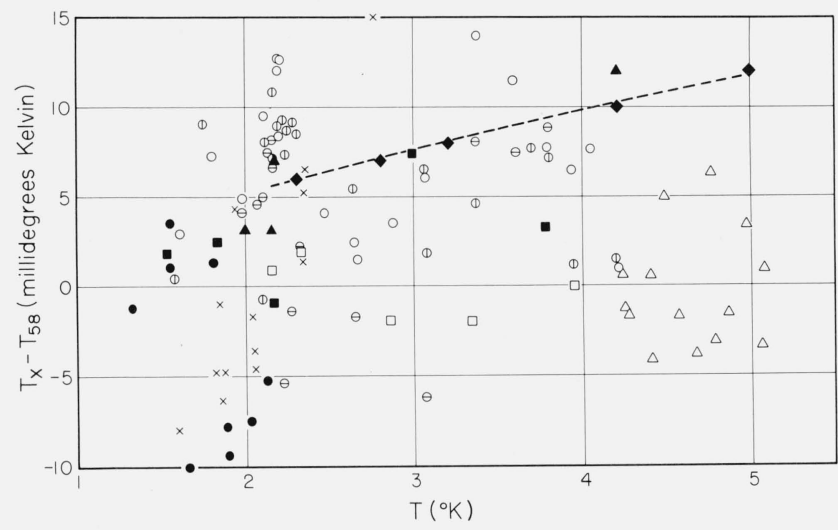

FIGURE 2. A comparison with $\mathrm{T}_{58}$ of temperatures derived from isotherm measurements, gas thermometry and the acoustical interferometer.

D $\ominus$ ○-Schmidt and Keesom [9] (gas thermometer. 1937).

- Kistemaker [10] (gas thermometer. 1946). - Kistemaker [10] (isotherms. 1946).

- Berman and Swenson [11] (gas thermometer. 1954)

- Keller [12] (helium 4 isotherms. 1955).

- Keller [12] (helium 3 isotherms. 1955).

-Preliminary results [4.5] (velocity of sound in helium 4 gas, acoustical interferometer. 1962

-Present work (isotherms determined by the NBS acoustical thermometer). not prepared to present a value for the absolute accuracy of our values of temperature, if one believes that the $T_{58}$ scale is smooth with respect to the true thermodynamic temperature scale, the plot suggests that perhaps the present data are the most self consistent of any temperature measurements that have been made below $5{ }^{\circ} \mathrm{K}$. (The dispersion in temperature values resulting from our different methods of isotherm analyses indicates that our deduced values of temperature are not critically dependent upon a particular method of analysis.)

The reproducibility of points on an isotherm appears to be within $\pm 0.002{ }^{\circ} \mathrm{K}$ although it is obvious that most of the isotherms need a better definition, that is, a greater number of points. Nevertheless, we do feel that the indicated conclusions of our measurements are important enough to be presented now rather than be delayed until the complete program in this temperature range has been finished.

\section{References}

11] NBS Tech. News Bull. 47. 175 (1965); NBS Misc. Publ. 253 (Nov. 1963).

[2] F. Everling. L. A. König. H. E. Mattuch, and A. H. Wapstra, Relative nuclidic masses. Nuclear Phys. 18, 529 (1960).

[3| J. L. and E. S. Stewart. J. Acoust. Soc. Am. 24, 22 (1952); M. Greenspan. Physical Acoustics 2A, 30 (Academic Press. Inc.. New York. 1965).

[4] G. Cataland. M. Edlow, and H. H. Plumb, Temperature, Its Measurement and Control in Science and Industry, C. M. Herzfeld, ed., 3, F. G. Brickwedde, ed., Pt 1 (Reinhold Publishing Corp., New York, 1962).

[5] G. Cataland and H. H. Plumb, J. Acoust. Soc. Am. 34, 1145 (1962).

[6] G. Cataland and H. H. Plumb, Proceedings of the Eighth International Conference on Low Temperature Physics (But terworths, Washington, 1963).

[7] F. G. Brickwedde, H. van Dijk, M. Durieux, J. R. Clement. and J. K. Logan, J. Research NBS 64A (Phys. and Chem.) No. $1.1(1960)$.

[8] H. van Dijk, Progress in Cryogenics, p. 123 (Heywood and Com pany. Ltd.. London, 1960).

[9] G. Schmidt and W. H. Keesom, Leiden Commun. No. 250b: Physica 4, 963 (1937).

[10] J. Kistemaker and W. H. Keesom, Leiden Commun. No. 269b: Physica 12, 227 (1946).

[11] R. Berman and C. A. Swenson. Phys. Rev. 95, 311 (1954).

[12] W. E. Keller, Phys. Rev. 97, 1 (1955); Phys. Rev. 100. 1790 (1955).

(Paper 69A6-375) 